\title{
Polyoxometalate based soft chemical route for preparation of Pt nanorods and self-assemblies
}

\author{
S SHANMUGAM, B VISWANATHAN* and T K VARADARAJAN \\ Department of Chemistry, Indian Institute of Technology Madras, Chennai 600 036, India
}

MS received 17 January 2005

\begin{abstract}
A soft chemical route is described for the preparation of platinum nanorods and self-assemblies over photochemically reduced polyoxometalate (silicotungstate) containing composite films. Transmission electron microscopy shows that the diameters of the platinum nanorods are around 55-60 $\mathrm{nm}$. The formation of platinum nanorods on solid-liquid interface reactions was explained on the basis of single site growth mechanism and diffusion limitation aggregation process.
\end{abstract}

Keywords. Polyoxometalates; platinum nanorods; diffusion limitation aggregation; sol-gel.

\section{Introduction}

Metal nanoparticles have received attention in recent years because of their potential applications in microelectronics (Schmid 1992; Andres et al 1996), photocatalysis (Kamat 2002), magnetic devices (Thomos 1988) and powder metallurgy (Perenboom et al 1981). The intrinsic properties of a metal nanoparticle are mainly determined by size, shape, composition, crystallinity and morphology (Dnruxm et al 1999; Dickson and Lyon 2000). In principle, one could control these parameters by adopting various synthetic strategies and in recent years, several approaches have been considered. Typical ones include using channels in macroporous membranes (Martin et al 1999), mesoporous materials (Han et al 2000), carbon nanotubes (Ugarte et al 1996) and DNA chains (Braun et al 1998). Although many successful approaches, such as vapour-solid and liquid-solid phase methods exist for the fabrication of $1 \mathrm{D}$ nanomaterials and their arrays, the widely used method is still chemical or electrochemical deposition directed by an appropriate porous "hard" template such as alumina membranes, diblock copolymers, carbon nanotubes and zeolite/mesoporous silica (Sloan et al 1999; Zhang et al 1999; Huang et al 2000, 2002). The hard template approach is an effective route to prepare metal and semiconductor $1 \mathrm{D}$ nanomaterials. However, most of the templates are tedious to prepare and dissolution of the template requires corrosive media.

Soft chemical methods have been employed for the synthesis of noble metal sols (Toshima and Takahashi 1992; Duff et al 1995; Attard et al 1997; Thurn-Albrecht et al 2000; Chen and Kimura 2001; Pol et al 2003) which in-

*Author for correspondence (bvnathan@iitm.ac.in) volve the reduction of relevant metal salt in the presence of a suitable stabilizing medium, which is useful to control the particle size and shape. Polyoxometalates (POM) are well-defined metal-oxygen cluster anions. Recently, polyoxometalate has been employed as reducing, stabilizing agent for the preparation of noble metal nanoparticles in homogeneous medium (Troupis et al 2002; Mandal et al 2003).

In this communication, a method for the preparation of platinum nanorods and self-assemblies of nanorods using reduced silicotungstate-based composite at room temperature is demonstrated. The method consists of solidliquid interface reaction (heterogenous) where metal ions interact with the active components and get reduced at the interface giving rise to specific shapes.

\section{Experimental}

UV-visible spectra were obtained by Perkin-Elmer Lamda 17 spectrophotometer. Transmission electron microscopy (TEM) images were taken using Philips CM12/STEM, Scientific and Analytical Equipment. TEM sampling grids were prepared by placing $2 \mu$ l of the solution on a carbon-coated copper grid and the solution was evaporated at room temperature.

The organic-inorganic composite was prepared by solgel method. For a typical synthesis, $1.5 \mathrm{~g}$ of polyvinyl alcohol (PVA 72000) was dissolved in $30 \mathrm{ml}$ distilled water stirred for $30 \mathrm{~min}$ and $2.5 \mathrm{ml}$ of tetraethylorthosilicate was added. The mixture was stirred for $10 \mathrm{~min}$, and then $0.5 \mathrm{~g}$ of silicotungstic acid was added and refluxed at $343 \mathrm{~K}$ for $6 \mathrm{~h}$. The final transparent solution was coated on glass slide by spin coating method. This was dried in oven at $50^{\circ} \mathrm{C}$ for $10 \mathrm{~min}$ and irradiated in sunlight for $60 \mathrm{~min}$. 


\section{Results and discussion}

The photoreduction of silicotungstic acid was monitored through UV-visible spectroscopy, which has a characteristic band around $750 \mathrm{~nm}$ indicating the formation of single electron reduced silicotungstate ion (figure 1). Up to $60 \mathrm{~min}$, with an increase in the irradiation time the intensity at $750 \mathrm{~nm}$ increased. After $60 \mathrm{~min}$ irradiation, there was no change in the absorption band $(750 \mathrm{~nm})$ indicating that all the silicotungstate ions in the composite have been reduced. The ESR spectra of photoreduced composite film exhibited a signal at $g=1.813$ at $77 \mathrm{~K}$, which is due to the formation of single electron reduced species, $\mathrm{SiW}_{12} \mathrm{O}_{40}^{5-}$ (Varga et al 1970). The photoreduced composite film was stable in air for one week and in inert atmosphere for three months (Viswanathan et al 2003). This reduced composite was dipped in beaker containing chloroplatinic acid $\left(5 \times 10^{-3} \mathrm{M}\right)$ solution. The reduction of metal ions and reoxidation of composite were monitored through UV-visible spectroscopy. The growth and formation of platinum nanorods were monitored through TEM.

The exact mechanism for the formation of platinum nanorods via this reduction technique is still under investigation. The proposed hypothesis is based on the electron microscopic and the absorption spectroscopic studies. Figure 2 shows the transmission micrographs of product obtained at different intervals of time. The formation of nanoparticles in the initial stage is evidenced from the TEM image shown in figure $2 \mathrm{a}$. The metal ions, which are in contact with the surface of the composite film, get reduced to give metal nanoparticles $(10-15 \mathrm{~nm})$. The slow dissolution of small platinum particles into the solution might also play a role to give anisotropic nanocrystals. The obtained nanoparticles aggregate to give anisotropic facets through the Ostwald ripening (Milligan and Morris 1964) (figure 2b). The inset shows the selected area electron diffraction (SAED) pattern for the facets which reveals that only a hexagonal diffraction spot pattern was observed, indicating the formation of single crystal platinum growing along in [111] direction. Beck et al (2000) reported a controlled growth of palladium particles at the solid/liquid interface on silica. The formation of anisotropic platinum nanocrystals at the solid-liquid interface indicates that the growth is highly localized, thus leading to considerable growth of the crystals away from the interface forming nanoparticles of triangular, hexagonal, pentagonal, square particles as evidenced from the TEM image (figure 2c). The anisotropic cluster particles grow into bigger particles through cluster-cluster aggregation (Sander 1986) giving rise to flower like morphologies (i.e. scaling is predominant) (figure $2 \mathrm{~d}$ ). The sharp parts of the structure grow faster than the rest of the structure (Witten and Sander 1981); it will catch random walkers more efficiently than the flat portions of the surface, thus giving rise to nanorods having a length of $350 \mathrm{~nm}$ and a diameter of $55 \mathrm{~nm}$ (figure 2e). Electron diffraction studies reveal that the platinum nanorods are highly single crystalline in nature.

The formation of platinum nanorods could be explained through the diffusion limited aggregation mechanism (DLA). In the present case, the reduction takes place in two-dimensional solid-liquid interface. At the initial stage, the platinum ions interact with the surface of the

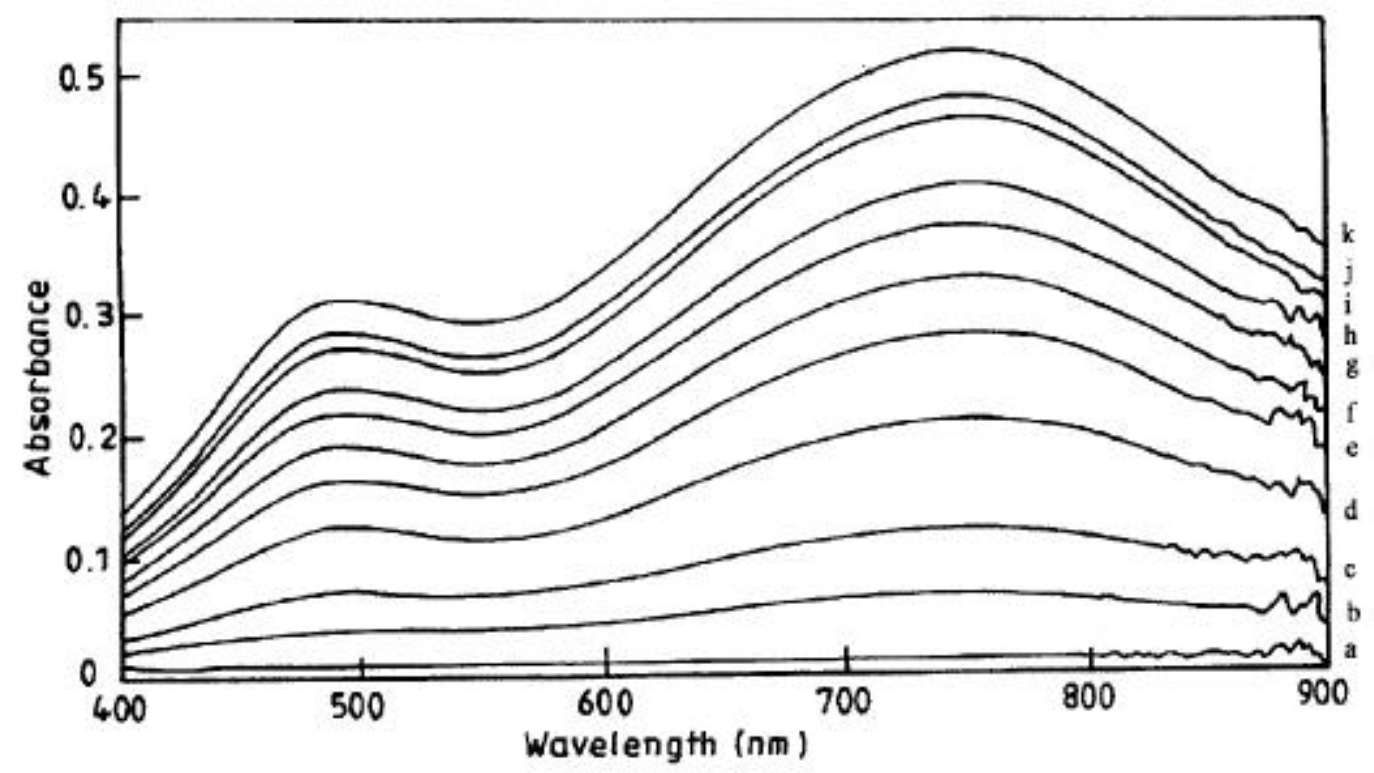

Figure 1. UV-visible absorption spectra of composite film irradiated in sunlight. a. 0, b. 5, c. 10, d. 15, e. 20 , f. 25 , g. 30 , h. 40 , i. 50 , j. 55 and k. 60 min. 

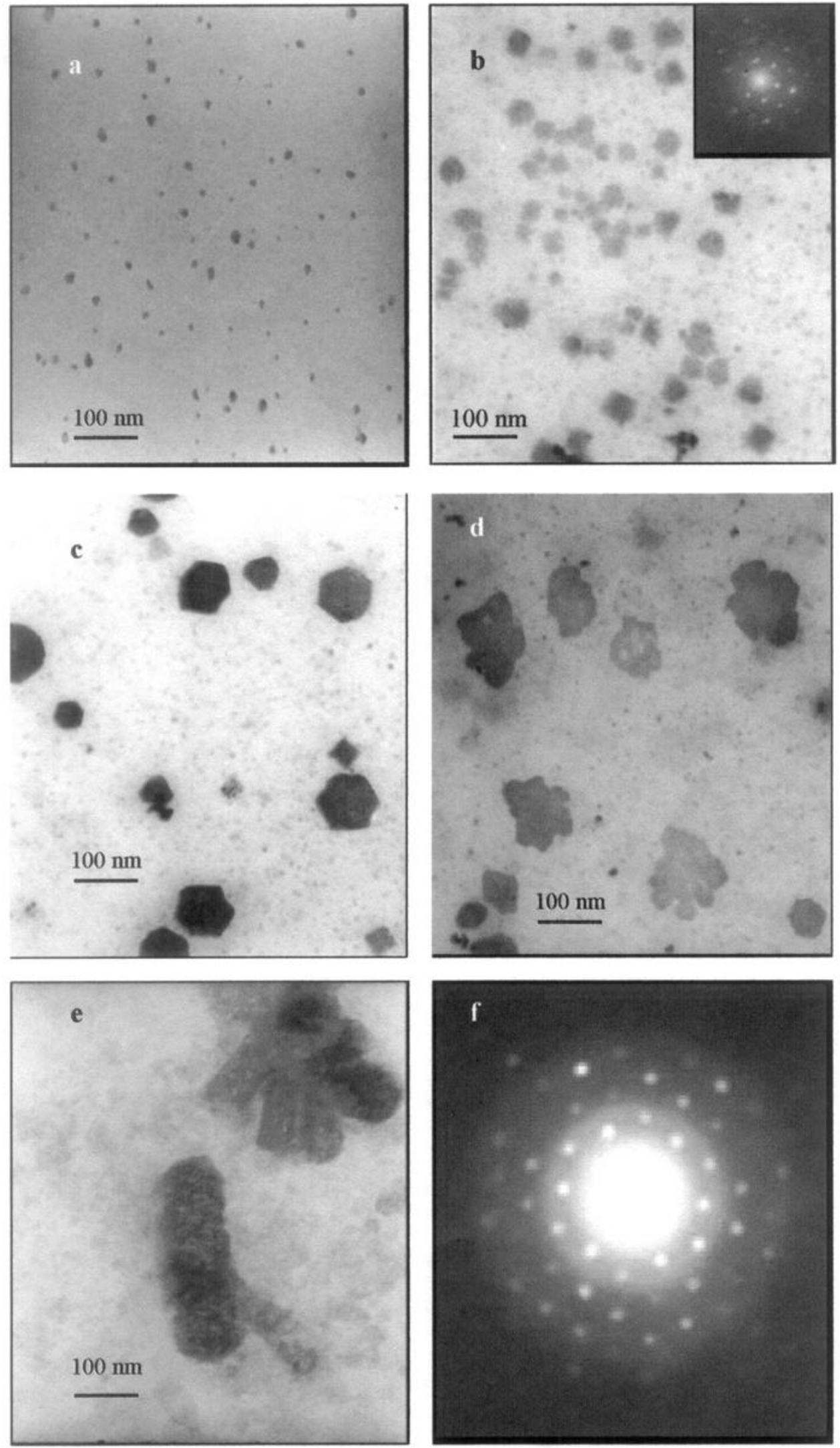

Figure 2. TEM image of reaction mixture at different intervals of time. a. 5, b. 10, c. 15, d. 20, e. $30 \mathrm{~min}$ and $\mathrm{f}$. electron diffraction pattern of figure 1e. 


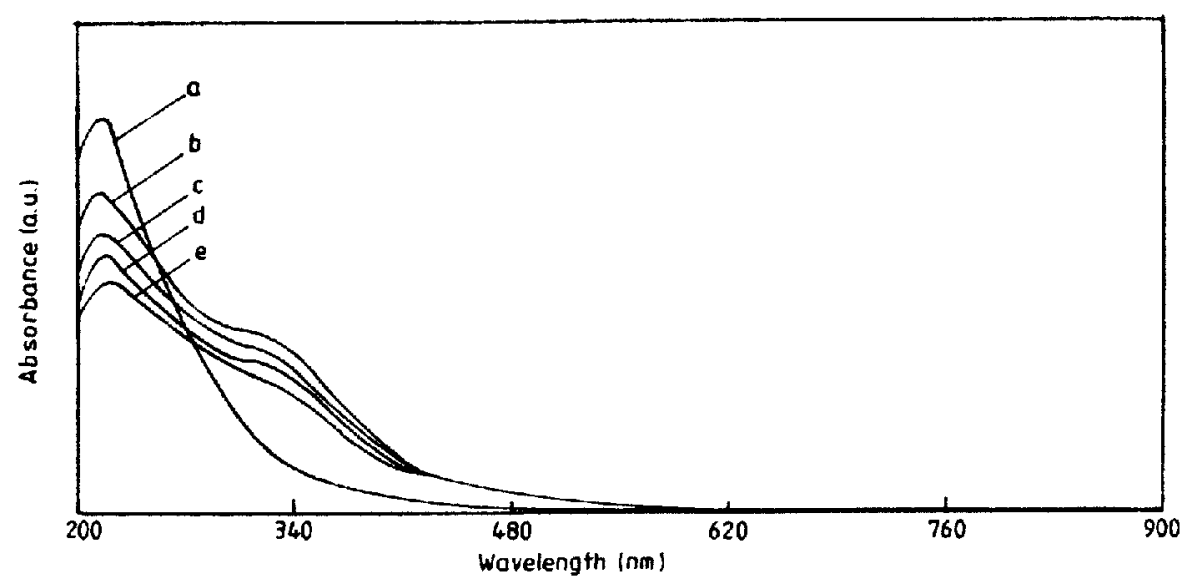

Figure 3. UV-visible absorption spectra of reaction mixtures at different time intervals. a. 0 , b. 5 , c. 10, d. 20 and e. $30 \mathrm{~min}$.

composite, get reduced and leave the surface. The new Pt nanoparticles, which are formed at the interface get stuck to the platinum particles and grow into bigger clusters (Zhang and Lagally 1997). In order to understand the formation of platinum nanorods by this method, controlled experiment was carried out. The composite gel (without making film) was irradiated in sunlight for $60 \mathrm{~min}$ and chloroplatinic acid was added (homogeneous reaction), which resulted in the disappearance of blue colour. The product obtained was mainly composed of irregular platinum nanoparticles without nanorods. This result indicates that composite film (solid-liquid interface) is vital for the formation of platinum nanorods.

Figure 3 shows the UV-visible absorption spectra of the reaction mixture taken at different time intervals $(0$, 5, 10, 20, $30 \mathrm{~min}$ ). Aqueous chloroplatinic acid has an absorption band at $220 \mathrm{~nm}$. It can be seen that the absorption band of $\mathrm{PtCl}_{6}^{2-}$ decreases as the reaction proceeds. At 5 th min, the appearance of a weak surface plasmon band at $340 \mathrm{~nm}$ indicates the formation of platinum nanoparticles with diameters of $10-20 \mathrm{~nm}$ in relatively low concentration. The absorption band of the platinum particles is mainly due to the interband transition from the ' $d$ ' band lying rather close to ' $s p$ ' band in the metal (Henglein et al 1995). For silver and gold nanoparticles, the red shift of surface plasmon band have been observed as the particles grow (Satoh et al 1994), but for the platinum nanoparticles (Weaver et al 1981), the surface plasmon band is weak and blue shifted. The intensity of the surface plasmon band decreases as the platinum particle size increases. When platinum forms clusters, the absorption spectrum becomes more flat which may be due to the dipole-dipole interaction of the aggregated particles (Quinten et al 1989). As the reaction proceeds, the intensity of the band at 340 as well as $220 \mathrm{~nm}$ further decreased in intensity indicating that the platinum particles are growing and giving rise to anisotropic shapes. This

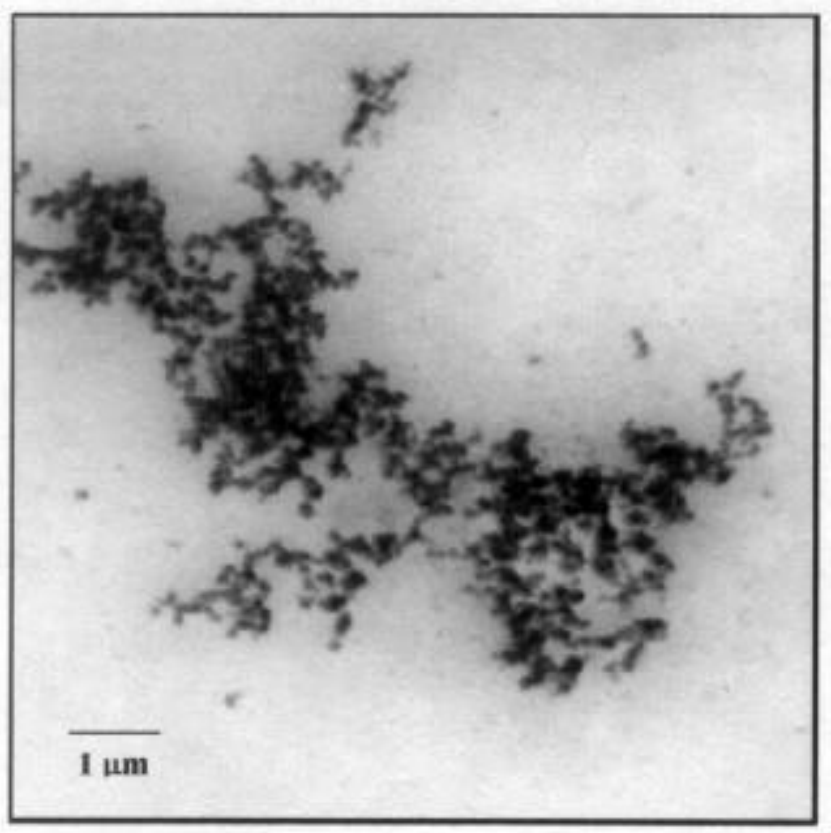

Figure 4. TEM image of the reaction mixture at $60 \mathrm{~min}$.

observation is well correlated with the TEM studies. As the reaction proceeds the spherical nanoparticles grow into nanorods through the intermediate structures. These experimental results suggest that it would be possible to control the particle morphologies of platinum by varying the experimental conditions (growth time).

Figure 4 shows the TEM image of the reaction mixture at $60 \mathrm{~min}$. Platinum nanorods are aggregated by DLA diffusion limited aggregation mechanism resulting in fractal like structure growth in which one nanorod after the other formed diffuses and sticks to the growing structure. From the TEM and absorption studies it has been speculated that platinum nanorods at the solid-liquid interface formed and the stepwise growth is shown in scheme 1 . 


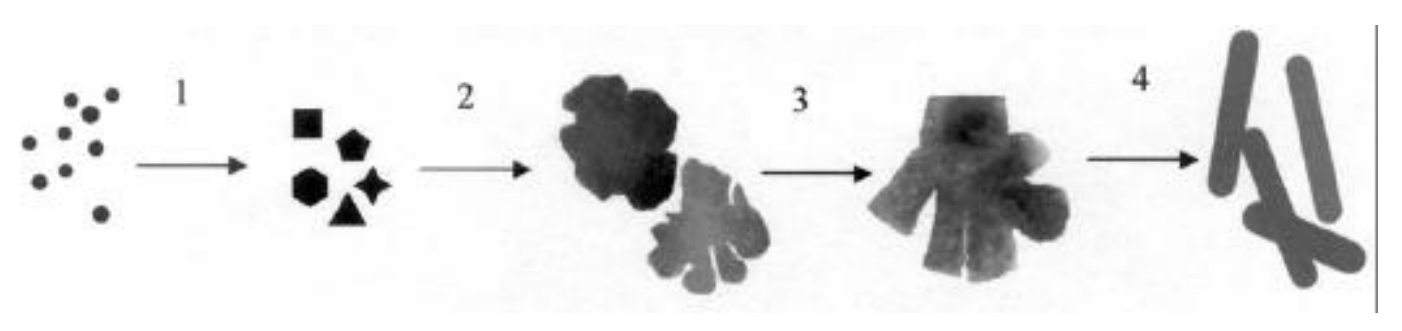

Scheme 1. Stepwise growth and formation of platinum nanorods.

In step 1 the formation of platinum nanoparticles are facile. The formed nanoparticles are reactive and will form facets in order to reduce the surface energy through the Ostwald ripening process (step 2). The cluster-cluster aggregation can be possible between the clusters of similar masses giving rise to three-dimensional growth and fragmentation leads to nanorods (steps 3 and 4).

\section{Conclusions}

In conclusion, a simple method has been described to prepare platinum nanorods. The adopted method does not require any stabilizing or polymer template to form platinum nanorods. The formation of platinum nanorods and self-assemblies is explained through the diffusion limited aggregation process. The growth and formation of platinum nanoparticles and self-assemblies were proposed based on TEM and absorption studies.

\section{References}

Andres P R, Bielefeld J D, Henderson J I, Janes D B, Kolagunta V R, Kubiak P C, Mahoney J W and Osifchin G R 1996 Science 2731690

Attard S G, Goltner G C, Corker M J, Henke S and Templer H R 1997 Angew. Chem. Int. Ed. Engl. 361315

Beck A, Horvath A, Szucs A, Schay Z, Horvath E Z, Zsoldos Z, Dekany I and Guczi L 2000 Catal. Lett. 6533

Braun E, Eichen Y, Sivan U and Ben-Yoseph G 1998 Nature 191775

Chen S and Kimura K 2001 J. Phys. Chem. B105 5397

Dickson M R and Lyon A L 2000 J. Phys. Chem. B104 6095

Dnruxm Y, Bastiaansen C, Caseri W and Smith P 1999 Adv. Mater. 11223

Duff G D, Edwards P P and Johnson G F B 1995 J. Phys. Chem. 9915934

Han J Y, Kim M J and Stucky D G 2000 Chem. Mater. 122068
Henglein A, Ershov G B and Malow M 1995 J. Phys. Chem. 99 14129

Huang H M, Choudrey A and Yang D P 2000 Chem. Commun. 1063

Huang M L, Wang T H, Wang B Z, Mitra A, Bozhilov N K and Yan S Y 2002 Adv. Mater. 1461

Kamat V P 2002 J. Phys. Chem. B106 7729

Mandal S, Selvakannan R P, Pasricha R and Murali Sastry 2003 J. Am. Chem. Soc. 1258440

Martin R B, Dermody J D, Reiss D B, Fang M, Lyon A L, Natan J M and Mullouk E T 1999 Adv. Mater. 111021

Milligan O W and Moriss H R 1964 J. Am. Chem. Soc. 863461

Perenboom J A A J, Wyder P and Meier P 1981 Phys. Rep. 78173

Pol G V, Gedanken A and Calderon-Moreno J 2003 Chem. Mater. 151111

Quinten M, Schonauer D and Kreibig U 1989 Z. Phys. D12 521

Sander M L 1986 Nature 322789

Satoh N, Hasegawa H, Tsujii K and Kimura K 1994 J. Phys. Chem. 982143

Schmid G 1992 Chem. Rev. 921709

Sloan J, Wright M D, Woo G H, Bailey S, Brown G, York E P A, Coleman S K, Hutchison L J and Green H L M 1999 Chem. Commun. 699

Thomos M 1988 J. Pure Appl. Chem. 60323

Thurn-Albrecht T et al 2000 Science 2902126

Toshima N and Takahashi T 1992 Bull. Chem. Soc. Jpn 65400

Troupis A, Hiskia A and Papaconstantinou E 2002 Angew. Chem. Int. Ed. Engl. 411911

Ugarte D, Chatelain A and de Heer A W 1996 Science 274 1897

Varga M G, Papaconstantinou E and Pope T M 1970 Inorg. Chem. 9662

Viswanathan B, Varadarajan K T and Shanmugam S 2003 Indian Patent File No: 222/MAS/2003

Weaver H J, Krafka E, Lynch W D and Koch E E 1981 Optical properties of metals (Karlsruhe: Fachinformationszentrum) Vol. 2

Witten T and Sander L 1981 Phys. Rev. Lett. 471400

Zhang Z and Lagally G M 1997 Science 276377

Zhang B Z, Gekhtman D, Dresselhaus S M and Ying Y J 1999 Chem. Mater. 111659 